\title{
Interpreting Prevotella and Bacteroides as biomarkers of diet and lifestyle
}

\author{
Anastassia Gorvitovskaia', Susan P. Holmes ${ }^{2^{*}}$ (D) and Susan M. Huse ${ }^{3}$
}

\begin{abstract}
Background: In a series of studies of the gut microbiome, "enterotypes" have been used to classify gut microbiome samples that cluster together in ordination analyses. Initially, three distinct enterotypes were described, although later studies reduced this to two clusters, one dominated by Bacteroides or Clostridiales species found more commonly in Western (American and Western European) subjects and the other dominated by Prevotella more often associated with non-Western subjects. The two taxa, Bacteroides and Prevotella, have been presumed to represent consistent underlying microbial communities, but no one has demonstrated the presence of additional microbial taxa across studies that can define these communities.

Results: We analyzed the combined microbiome data from five previous studies with samples across five continents. We clearly demonstrate that there are no consistent bacterial taxa associated with either Bacteroides- or Prevotella-dominated communities across the studies. By increasing the number and diversity of samples, we found gradients of both Bacteroides and Prevotella and a lack of the distinct clusters in the principal coordinate plots originally proposed in the "enterotypes" hypothesis. The apparent segregation of the samples seen in many ordination plots is due to the differences in the samples' Prevotella and Bacteroides abundances and does not represent consistent microbial communities within the "enterotypes" and is not associated with other taxa across studies. The projections we see are consistent with a continuum of values created from a simple mixture of Bacteroides and Prevotella; these two biomarkers are significantly correlated to the projection axes. We suggest that previous findings citing Bacteroides- and Prevotella-dominated clusters are the result of an artifact caused by the greater relative abundance of these two taxa over other taxa in the human gut and the sparsity of Prevotella abundant samples.

Conclusions: We believe that the term "enterotypes" is misleading because it implies both an underlying consistency of community taxa and a clear separation of sets of human gut samples, neither of which is supported by the broader data. We propose the use of "biomarker" as a more accurate description of these and other taxa that correlate with diet, lifestyle, and disease state.
\end{abstract}

Keywords: Microbiome, Biomarkers, Enterotypes, Human gut

\section{Background}

The gut microbiome plays an important role in human health and disease. Understanding what constitutes a health-promoting (eubiotic) or disease-promoting (dysbiotic) microbial community has become the focus of significant research. In 2011, Arumugam et al. [1] postulated that all human gut microbiomes could be classified as one of three distinct bacterial communities or "enterotypes" ${ }^{\prime 1}$. The researchers used ordination analysis on three

\footnotetext{
* Correspondence: susan@stat.stanford.edu

${ }^{2}$ Sequoia Hall, Stanford University, Stanford, CA, USA

Full list of author information is available at the end of the article
}

datasets: a set of 33 European, Japanese, and American subjects, a set of 85 Danish, and a set of 154 Americans. Each dataset independently displayed a similar clustering pattern with subjects dominated by one of three different taxa: Bacteroides, Provetella, or members of the order Clostridiales. The authors speculated that if this clear separation of clusters were evident across all human gut samples, people could be classified by their enterotype, which could in turn be used to guide diagnostics and treatment options. Only one of datasets showed clear separation between the Bacteroides- and Clostridiales-dominated samples, while two showed overlapping points consistent with a gradient. 
Since the introduction of the enterotypes hypothesis, several studies have evaluated their presence in other human gut communities. The first such study by $\mathrm{Wu}$ et al. [2] sequenced 98 Americans and used multiple clustering techniques but found only two of the three original clusters: Bacteroides and Prevotella, laying at either end of a Prevotella-Bacteroides gradient. The third cluster was not distinct in their data, with Ruminococcus (a Clostridiales genus) equally abundant in both of the first two enterotypes. They compared their findings with a previously published study on Italian and rural African children wherein Italian children had Bacteroides-dominated gut microbiomes and African children Prevotelladominated gut microbiomes. As part of our own analysis of over 200 healthy American subjects enrolled in the Human Microbiome Project (HMP) [3], we confirmed the results of $\mathrm{Wu}$ et al. that two of the three clusters were not distinct. Samples high in Bacteroides or members of the order Clostridiales, including Ruminococcus, form a continuum, rather than discrete clusters. While the Prevotella-dominated samples did cluster separately with the HMP data, we speculated that as more samples and studies were evaluated, the separation between clusters would diminish, and gut communities would appear as a continuum of abundance gradients between Bacteroides, Prevotella, and various members of the order Clostridiales. In a review article published soon after, Jeffrey et al. [4] reached the same conclusion that a gradient of microbial populations was a more accurate interpretation of the data.

Several studies thereafter sought to re-examine enterotypes using more careful clustering analyses [5-7]. Overall, these additional studies demonstrated that with increased statistical rigor, support remained for only two community types: one dominated by Prevotella and one dominated by Bacteroides or members of the Firmicutes [5]. Additionally, the utility of enterotypes to classify patients was shown to be limited given that individuals can shift between Bacteroides/Firmicutes- and Prevotelladominated communities over time, with as many as $30 \%$ of samples in one dataset shifting between enterotypes [6, 7]. Knights et al. [6] further cautioned that supervised ordination methods can lead to false clustering when the number of features in the data (here microbial genera) is much higher than the number of samples, as is the case in most human gut microbiome studies. Overall, these studies found that the weight of evidence did not support the classification of human gut microbiomes into discrete and stable enterotypes.

Despite the ongoing refutation of the appropriateness and stability of enterotypes, researchers continue to use them to interpret ordination analyses that separate Prevotella-dominated communities from those dominated by Bacteroides, Clostridiales, and a fourth taxon,
Bifidobacterium. These communities recur across various study populations and diets. For instance, in a study that predated the enterotypes hypothesis, De Filippo et al. [8] studied 29 children, 15 from Italy and 14 from Burkino Faso. The gut microbiomes of the Burkino Faso children were mostly dominated by Prevotella, while the Italian children were mostly dominated by Bacteroides and Clostridiales. Both populations included a few subjects dominated by Actinobacteria, mainly Bifidobacterium, a beneficial microbe associated with milk-based diets such as breast-feeding [9]. The Burkino Faso diet was rich in vegetables and fiber, and children were breast-fed up to 2 years of age. The diet of the Italian children was a "Western diet" low in fiber, incorporating more animal protein, fat, and sugar, and children tended to be breast-fed for only 1 year. It was postulated that diet could be a driving factor in shaping the gut microbiome.

Ou et al. [10] compared twelve African Americans and twelve native Africans as part of a study examining the higher prevalence of colon cancer in Americans. The populations clustered distinctly, with the African Americans dominated by Bacteroides and the native Africans by Prevotella. The native African diet was high in complex fiber, while the African American diet was a Western diet high in protein. Yatsunenko et al. [11] compared gut microbiome samples of healthy adults and children from Malawi, Venezuela, and the USA. They found a gradient of Bacteroides and Prevotella in both adults and children over 6 months. They also found a discrete group of samples dominated by Bifidobacterium, in children under 6 months of age, which is consistent with its association with breast-feeding. The US subjects clustered separately from the Malawian and Venezuelans, who had relatively more Prevotella.

In a comparison of 50 metropolitan and 46 rural Russians, Tyakht et al. [12, 13] found between two and three community types. Prevotella accounted for one of the clusters and was prevalent in samples from one of the rural villages, but Firmicutes (rather than the combination of Bacteroides and Firmicutes) and Bifidobacterium dominated the remaining samples. The ingredients of the diet eaten by the metropolitan subjects were similar to those in a Western diet, but the sources of their food were very different. The authors speculated that the lack of Bacteroides-dominated samples could be due to the metropolitan Russian food sources, which were homegrown rather than industrially processed. Overall, the results from these various studies are consistent. The gut microbiomes of Americans and Europeans having a Western diet tend to be dominated by Bacteroides and Clostridiales, while rural populations with a high fiber, low-protein diet tend to be dominated by Prevotella. 
Despite the frequent use of the term "enterotype" to describe clustering of the gut microbiomes of research subjects from different cultures and with different dietary habits, the membership of the microbial communities associated with the enterotypes has not been clearly defined. Prevotella, Bacteroides, Clostridiales, and Bifidobacterium are recurring taxa, but a deeper understanding of the community structure of samples dominated by these taxa across continents and cultures has not been undertaken. Whether distinct community types or gradients, stable over time or not, we do not have a functional description of what other bacteria comprise the Prevotella- or Bacteroides/Clostridiales-dominated clusters beyond the dominant taxon. Here we present an analysis of the combined data from these published studies to characterize the broader community membership of the two primary enterotypes, so that we might better understand what these communities can tell us about the human gut microbiome.

\section{Results and discussion}

Our analysis compiled 747 samples from five studies encompassing 484 genera. We included 126 samples from the Arumugam et al. [14]: 41 mixed Europe and Asia, 85 Europeans, 24 Native African and African American samples [10], 96 urban and rural Russians samples [12], 210 samples of healthy American adults from the Human Microbiome Project [15], and 291 adult samples from Malawi, Venezuela, and America [11].

\section{Multivariate analysis}

The "enterotype" hypothesis was initially proposed based on clustering results seen in a principal coordinates analysis. In our initial ordination analysis, we found that all three populations from the Yatsunenko study clustered separately from the remaining studies (Additional file 1: Figure S1). We therefore performed our primary multivariate analyses without these samples but then confirmed our findings with an independent analysis of them. Combined samples from the four remaining datasets do not cluster discretely with either metric or nonmetric multidimensional scaling $\left(\mathrm{PCoA}^{2}\right.$ or $\left.\mathrm{NMDS}^{3}\right)$, using Bray-Curtis or Morisita-Horn distances, but instead show a gradient across both dominant taxa and study populations (Fig. 1, Additional file 2: Figure S10). Coloring by dominant taxa (Fig. 1a) and the Prevotella ratio (the ratio of Prevotella to the sum of Prevotella and Bacteroides) (Fig. 1b) illustrate the relationship of axes 1 and 2 to Bacteroides (toward the upper right) and Prevotella (toward the lower left) in the samples. When colored by population, we see that the second axis separates the samples within a population, as does the first axis but to a lesser extent (Fig. 1c). In PCoA analysis of the Malawi, Venezuela, and US populations from the
Yatsunenko study (Additional file 3: Figure S7), the first axis appears to correspond primarily to the Prevotella ratio. The three countries are spread along the second axis with the US samples separating from the other two, but the Malawian and Venezuelan samples are intermingled. In general, these data do segregate along the first axis, with fewer intermediate samples than in the combined studies, but distinct clustering is unclear. These ordination results show that the primary axes continue to correlate with Bacteroides and Prevotella when the studies are combined.

To assess the persistent clustering of the microbial communities independent of the relative abundances of Bacteroides and Prevotella, we removed these two taxa from all the samples and reran the PCoA analysis (Fig. 1d, Additional file 3: Figure S7B). Without Prevotella and Bacteroides, the original microbial community classifications ("enterotypes") no longer segregate the samples. The ellipses in Figs. 1a and 1d were constructed as confidence regions for each of the groups; these regions would represent $95 \%$ of the points if the bivariate data were Gaussian. This demonstrates the effect on the clustering of removing the two dominant taxa. When the dominant taxa are included, the ellipses abut without overlapping. When the dominant taxa are removed, two of the ellipses are almost completely within the third, and all but three of the Prevotella-dominated samples are contained within the ellipse delineating the central Bacteroides samples. Separation of the original clusters is no longer present, and the remaining taxa now account for only $33 \%$ of the variability along axes 1 and 2, as opposed to $54 \%$. The ordinations of the full samples appear to be driven primarily by the relative quantities of these two highly abundant taxa and not by the presence of distinct microbial communities associated with each group. Interestingly, there is still clustering structure and differentiation of samples visible in Fig. 1d, as one would expect when comparing samples from patients in different geographic regions having different lifestyles and diets. These data structures, however, no longer correlate clearly with Bacteroides and Prevotella.

\section{Taxonomic components of microbial communities}

To identify taxa integral to a broader definition of microbial community structures, we looked for taxa that correlated with the dominant genus (Prevotella or Bacteroides) across studies (Additional file 4: Table S1). Several researchers reported correlations within Bacteroides-dominated samples within their studies, including Acidminococcus, Roseburia, Faecalibacterium, Anaerostipes, Parabacteroides, and Clostridiales [14], Alistipes only [16], Escherichia (Enterobacteriaceae) and Acinetobacter [10], and Faecalibacterium and Enterobacteriaceae [8]. None of these associations, however, were 


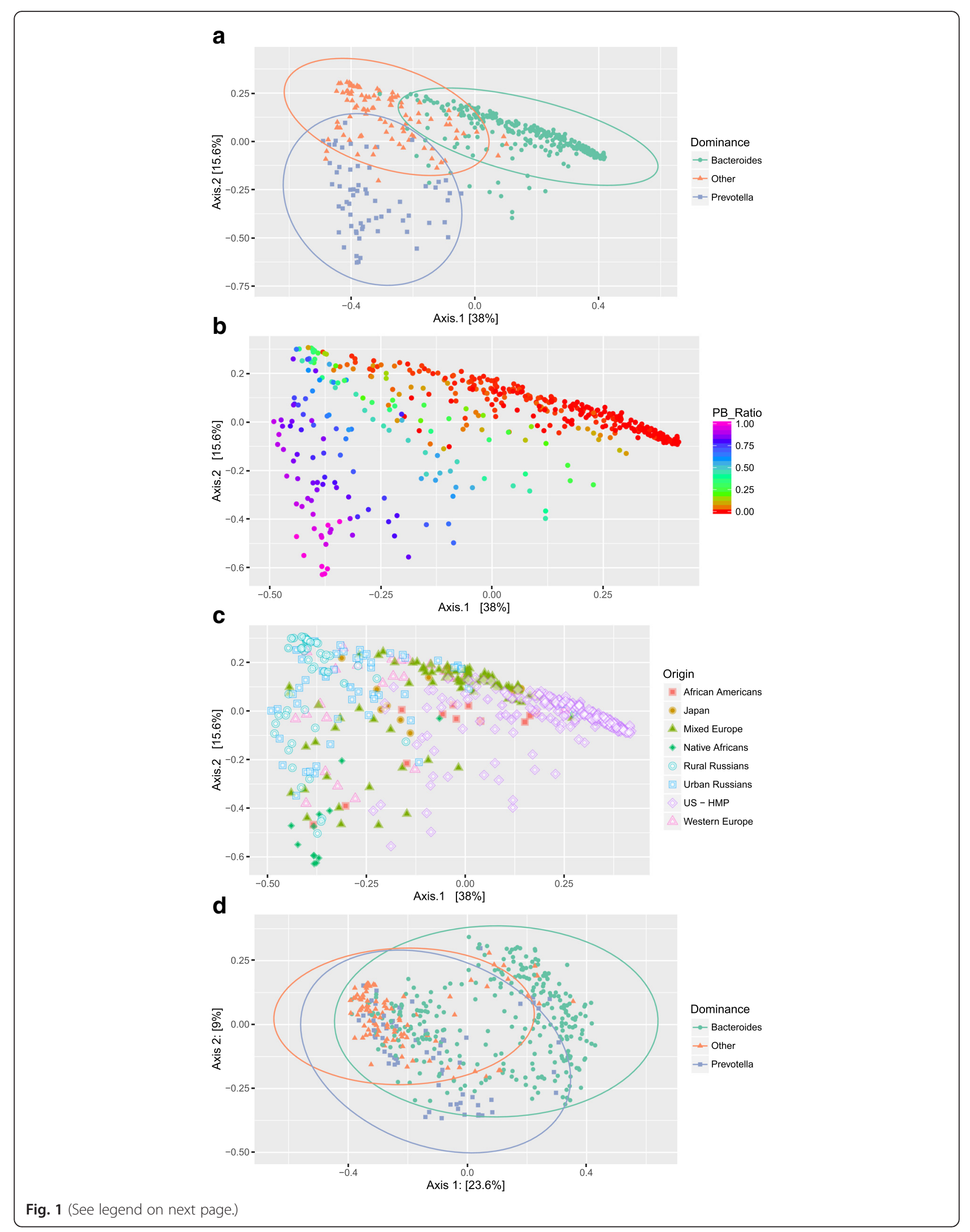


(See figure on previous page.)

Fig. 1 PCoA plots using the Bray distance metric with all the samples except for the Yatsunenko study. a Samples colored by their most prominent taxon. If the sample is dominated neither by Prevotella nor Bacteroides, it is classified as other. Ellipses were projected for each group in the plot. The ellipse axes represent the directions of the within-group covariance matrices, and their bounds represent two standard deviations in each direction from the cluster mean. $\mathbf{b}$ Samples are colored by their value for the Prevotella ratio (relative abundance of Prevotella/[Bacteroides + Prevotella]) on a spectrum with red indicating no Prevotella and purple no Bacteroides. c Samples are colored by population of origin. $\mathbf{d}$ The Bray distance has been recalculated without the relative abundances of Bacteroides and Prevotella. Samples are colored by most prominent taxon in the original samples distributions, and ellipses were projected for each group in the plot (same as in plot a)

apparent across all studies. In fact, only Faecalibacterium and Enterobacteriaceae appear in even two of the four studies that reported correlation analyses. No correlations were found within the Prevotella-dominated samples in more than one previous study. Prevotella was associated with Streptococcus, Enterococcus, Desulfovibrio, and Lachnospiraceae [14], Succinivibrio and Oscillospira [10], and Xylanibacter and Butyrivibrio [8]. The majority of taxa found in the Russian communities $[12,13]$ were not found in previously reported studies. Tyakht et al. used triplets of the three most abundant members of their various samples to compare with these other studies and found that $43 \%$ of their samples were dominated by triplets not found in the non-Russian groups.

To confirm this finding of no consistent multi-study taxa associated with either dominant taxon, and to increase our statistical power of detection, we combined the samples from all of the studies and reanalyzed them. We used the Spearman coefficient (a nonparametric method used in most of the previous studies) as well as SparCC [17]. Using Spearman on all the data and the DESeq2 [18] negative binomial test on the HMP data, we found no taxa in the combined set of Prevotelladominated samples that correlated with Prevotella above our threshold for statistical and biological significance (Additional file 4: Table S1). SparCC did find two Clostridiales genera that correlated with Prevotella in the combined dataset, Ruminococcus $\left(R^{2}=0.77\right)$ and Dialister $\left(R^{2}=0.73\right)$, but neither of these had a significant correlation in any single study. In the combined Bacteroides-dominated samples, we did not find any significant correlations using the Spearman correlation. A few Clostridiales taxa and one Bacteroidales genus in individual studies did seem correlated with Bacteroides, but none of these were found in more than two of the studies. Using SparCC, Subdoligranulum $\left(R^{2}=0.75\right)$ and Faecalibacterium $\left(R^{2}=0.73\right)$ correlated with Bacteroides but only in the combined set, not in any of the individual datasets. No taxa were significant in more than one of the studies when analyzed separately. These data do highlight that members of both Bacteroidales and Clostridiales are prominent members of the human gut microbiome of Americans and Western Europeans. To explore additional taxonomic differences between groups defined in the Yatsunenko and HMP studies as Prevotella-rich and Bacteroides-rich, we used the package DESeq2 and the tests based on variance transformed data using the negative binomial model (DESeq function with default arguments) as explained in [18]. This provided a ranking of the most differentially abundant taxa. The analysis of the Yatsunenko data showed Parabacteroides, Alistipes, and Subdoligranulum, to be more prevalent in Bacteroides-dominated samples, but these results were not reflected in the HMP data. The DESeq2 analysis confirmed the previous analyses, showing no taxa consistently associating with the Bacteroides- and Prevotella-dominated samples. Recent work by Lovell et al. [19] has emphasized the importance of proportionality analysis when working with relative abundance data. Applying their proposed algorithm on our compiled data, we still found no significant proportionalities across studies. The taxa that had proportionality statistics less than 0.01 were either very rare or even possibly artifacts of the sequencing process. Two of these taxa were found in only one sample, and all of the others were identified in only populations from one study (the European samples from the Arumugam study). They are not prevalent in either the Prevotella- or Bacteroides-dominated samples, and in fact, are absent from the majority of both. These taxa are clearly not important functional components or biomarkers of a human gut community. The analysis itself can be found in the Additional file 5 containing the Rmd commands, data and output. This file contains all the code the reader could require to follow the same workflow on a different data set.

To further understand groupings based on the abundance of Prevotella, we compared the Prevotella ratio (Prevotella/[Bacteroides + Prevotella $])$ for all samples in each study. Figure 2 illustrates the clear presence of samples across the full spectrum of relative abundances of Prevotella and Bacteroides, although different studies have varying numbers of samples in the intermediate ranges. Americans and Europeans, which make up the largest number of subjects, have fewer samples with intermediate Prevotella ratios, while studies containing more rural subjects have a greater number of intermediate samples. Bacteroides tends to be higher in Western populations, with a more even distribution of values across samples (Additional file 1: Figure S1), while Prevotella tends to have fewer intermediate values in the 


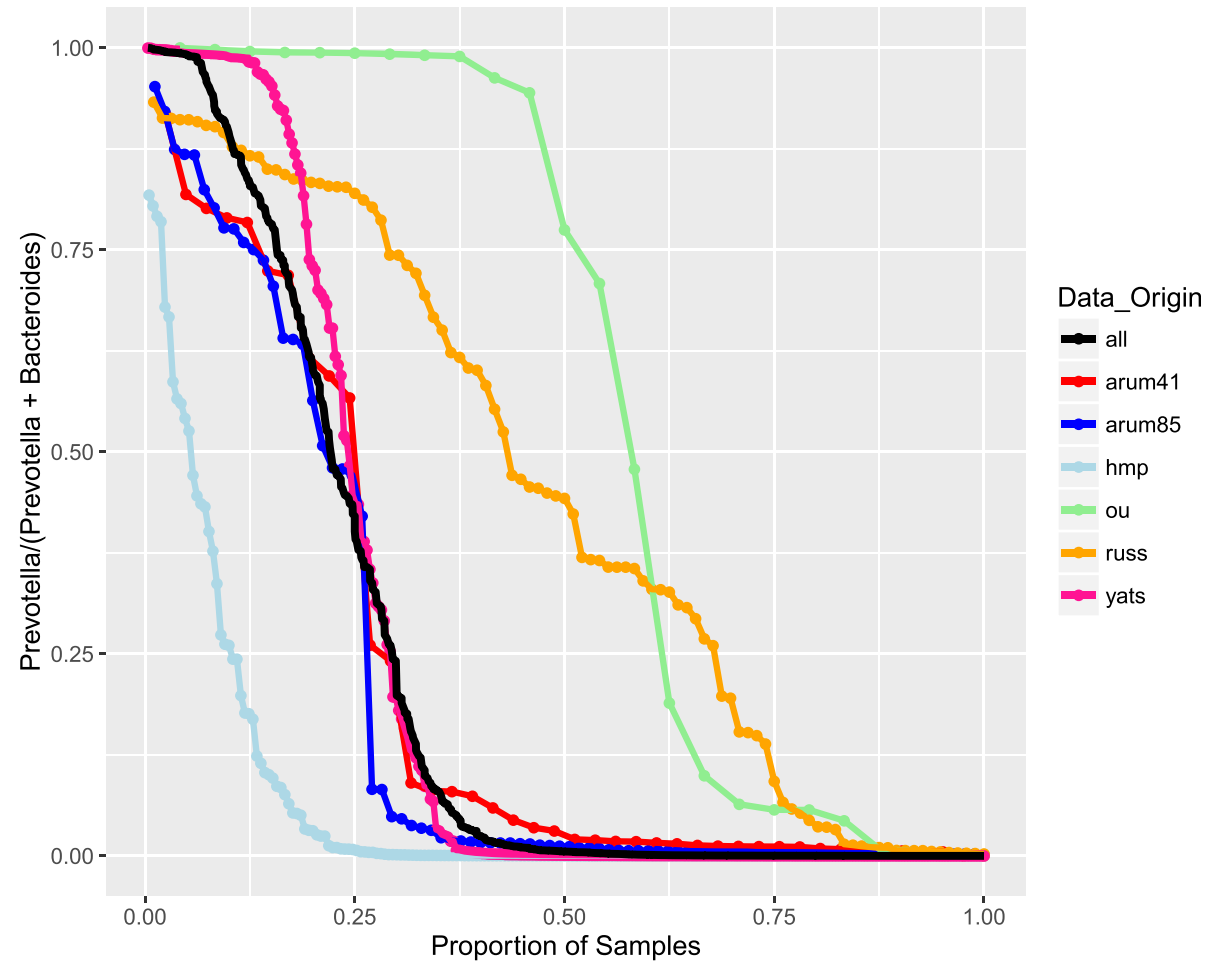

Fig. 2 Prevotella ratio distributions (relative abundance of Prevotella/[Bacteroides + Prevotella]) within each population. The x-axis represents quantiles (from 0 to 1) to facilitate simultaneous plotting. The black line represents all the samples. The green points are from the Native African vs. African American Ou et al. study, the yellow points are from the Russian Urban vs Rural Tyakht et al. study, the pink points are from the Malawi, Venezuela, US Yatsunenko et al. study, the red points are from the Mixed Europe and Asia Arumugam et al. study, the blue points are from the European Arumugam et al. study, and the light blue points are from the NIH Human Microbiome Project study

Western samples and more in Russian and rural samples (Additional file 6: Figure S2).

We compared the abundance distributions of Prevotella and Bacteroides with other common genera in the combined studies (Fig. 3 and Figure S11 in the Additional file 7 for the same boxplot without the Yatsunenko study). Bacteroides, Prevotella, Lachnospiraceae, Faecalibacterium, Roseburia, Ruminococcus, Alistipes, and Coprococcus are among the next most common genera across all studies. Bacteroides, Prevotella, and Alistipes are members of the order Bacteroidales, while the remaining taxa are all members of Clostridiales. In approximately $42 \%$ of samples, Bacteroides has a relative abundance between 10 and $40 \%$, with more than $80 \%$ of samples having a relative abundance greater than $5 \%$. Prevotella has a relative abundance between 10 and $40 \%$ in $16 \%$ of samples, but $75 \%$ of samples have a relative abundance less than $5 \%$. While Bacteroides exhibits a higher proportion of intermediate abundances overall than does Prevotella, Prevotella shows a spectrum of abundances rather than a bimodal distribution as some have assumed, with more samples between 40 and $80 \%$ than any taxon other than Bacteroides.

\section{Conclusions}

Although several studies show some discrete clusters, most other studies have shown a continuous gradient of communities [2-7] that can change within an individual over time [6, 7], and no one has evaluated the taxonomic composition of the clusters across studies. The consistent body of evidence accumulating about the structure and role of the microbiome in human health demonstrates that standard microbiome methods are sufficient to compare across studies of the human gut. Here we analyze the combined microbiome data of five earlier studies and show that the gut microbiomes labeled as Bacteroides and Prevotella "enterotypes" do not represent consistent or predictable communities. No other bacterial taxa correlate with either of the two primary biomarkers across studies other than the taxa for which they are named. While the original study included a correlation analysis, the results represented the differences in their particular study populations, rather than a set of universal correlations. As was pointed out as early as 1896 by Pearson [20], proportions give rise to spurious correlations and our approach here relies on the study of proportions that eliminate the common denominators as suggested in [19]. 


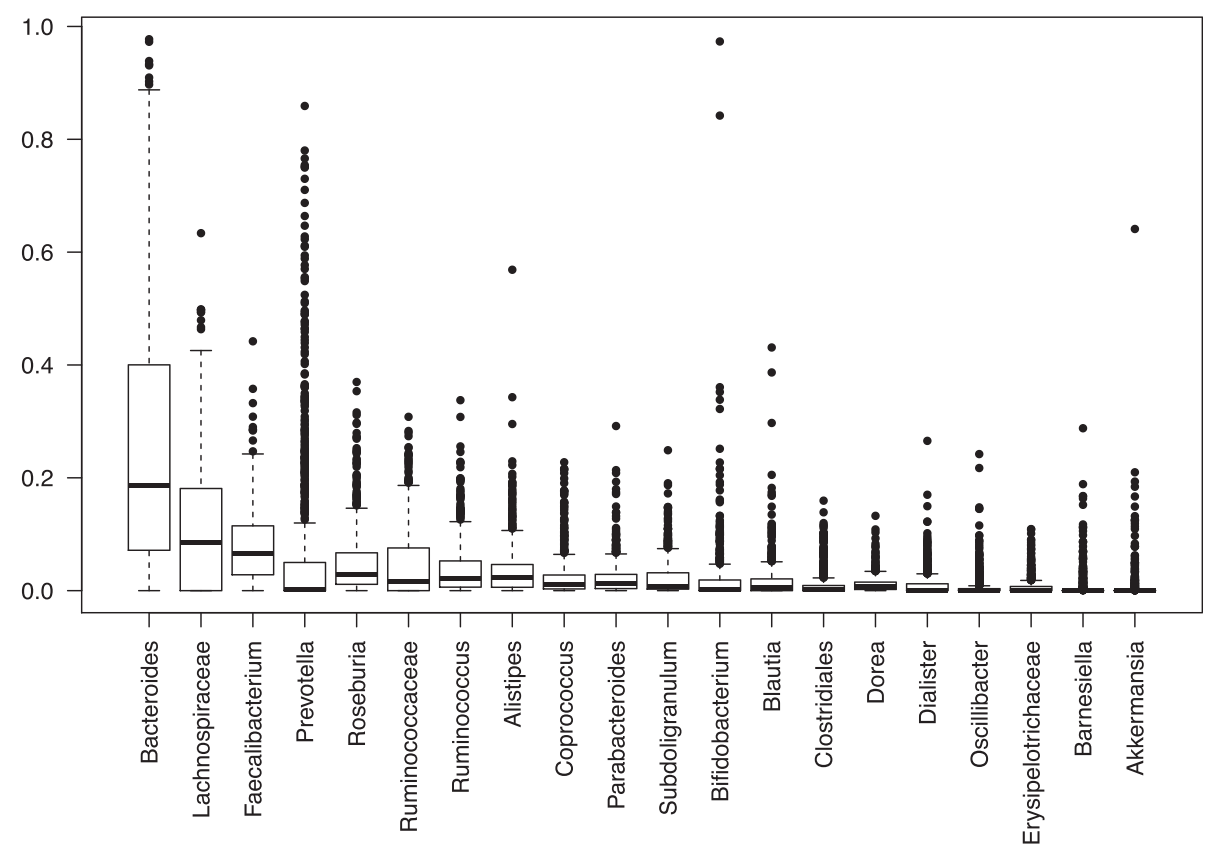

Fig. 3 Boxplot of the top 20 taxa across all the studies. The dark horizontal line represents the mean relative abundance, and the box represents the bounds of the 25th and 75th percentiles

Despite ongoing re-evaluation of the evidence, researchers have continued to use the term "enterotype" to describe differences between microbiome communities in study populations. The continued use of enterotype classifications is primarily a result of the ordination algorithms used to differentiate sets of samples based on the most distinguishing taxa. Of all the taxa in the combined studies, Bacteroides and Prevotella are the only two whose relative abundances frequently exceed $40 \%$ (Fig. 3). Additionally, it is often the case that when Bacteroides is high in a sample, Prevotella will be low, and vice versa. These two taxa, therefore, contribute more than any others to the pairwise dissimilarity between samples. The various ordination method (PCoA, NMDS) plot samples with larger pairwise distances farther apart, and samples with smaller pairwise distances closer together. Because Prevotella and Bacteroides have the greatest range of values and tend to be inversely related, they contribute the most to the magnitude of pairwise distances, and therefore contribute the most to separation in ordination plots, leading to Bacteroides- and Prevotella-dominated gradients in ordination plots. Bacteroides abundance correlates significantly with the first axis, having a correlation of 0.93 ( $p$ value $<10^{\wedge}-5$ ). Prevotella abundance correlates significantly with the second axis having a negative correlation of -0.8 ( $p$ value $\left.<10^{\wedge}-4\right)$.

To illustrate how these Bacteroides and Prevotella abundances can drive ordination plots and that no other taxa were associated to the "enterotype" definitions, we compared ordination results with and without these two taxa (Fig. 1a, d, Additional file 8: Figure S4D, Additional file 9: Figure S5D, Additional file 3: Figure S7D, and Additional file 10: Figure S9D). We found that the remaining taxonomic-community members did not continue to separate these samples by microbial component classification. Ordination plots are not a means for defining underlying community characteristics and can only be part of the exploration of community structure rather than the endpoint of analysis. The dearth of any consistent features of these communities across independent studies is a reminder for us not to extrapolate from PCoA and NMDS plots to assumptions about the defining characteristics of the entire communities. In studies comparing only two very different populations (e.g., Western urban and non-Western rural), separation will likely remain even without Bacteroides and Prevotella, but we cannot extrapolate from any two populations to consistent clustering across a range of diverse populations when the two dominant taxa, Bacteroides and Prevotella, are removed. The combination of correlation results and ordinations with and without the dominant taxa can be used to compare across other studies to interpret clusters as driven by the most dominant taxa or by a deeper community structure.

We believe that using the term "enterotype" to describe gut communities is misleading because it implies a microbiome community type shared among samples 
with the same enterotype designation. We propose the use of the term "biomarker" to describe the dominant taxon of the community rather than "enterotypes" to describe gut microbiomes. Biomarkers serve as a measurable indicator of a biological state or environmental exposure [21]. In this regard, genera such as Prevotella, Bacteroides, and Bifidobacterium potentially serve as effective biomarkers of diet and lifestyle. Across the studies, Prevotella was associated with non-Western, rural communities, and a plant-based diet rich in polysaccharides and fiber $[2,8,10,11,13,21]$. In each study sampling the gut microbiome of subjects from native or rural villages (Burkina Faso [8], South Africa [10], Venezuela and Malawi [11], and Russia [12]), Prevotella was commonly dominant in the adult samples. In these same studies, Bacteroides-dominated samples were more generally associated with samples from US and European subjects eating a Western diet, richer in protein and fat. Wu et al. [2] graphically illustrated correlations between Bacteroides and dietary amino acids and fats.

Beyond Prevotella and Bacteroides, additional taxa can also serve as biomarkers of diet or disease. Bifidobacterium was identified as a dominant bacterium in several studies $[8,11,12]$, especially the gut microbiome of children $[8,11]$. Bifidobacterium has specific adaptations that provide a competitive advantage for metabolizing the specific human milk oligosaccharides found in breast milk [9]. Various members of the phylum Firmicutes are associated with plant-derived fiber, amino acids, and fats. Firmicutes as a phylum, however, is unlikely to be an effective candidate for a biomarker, as it incorporates a diversity of genera. David et al. [22] report an increase in the bile-tolerant bacteria: Alistipes, Bilophila, and Bacteroides with an animal-based diet, while Roseburia, Eubacterium, and Ruminococcus were more abundant on a diet rich in plant polysaccharides. Some of these genera also have the potential to be biomarkers. Ou et al. [10] compared African Americans with a high risk of colon cancer and native Africans with only rare cases of colon cancer and suggested that Bacteroides, Escherichia, and Acinetobacter may be possible biomarkers for cancer. Baxter et al. [23] found correlations between Bacteroides, Parabacteroides, Alistipes, and Akkermansia with increased tumor burden and Clostridiales Cluster XIVa (which includes Roseburia and Faecalibacterium) with decreased tumor burden. Fusobacterium has also been associated with colon cancer [24]. The use of specific bacterial taxa to characterize the ecological roles of microbial community members was recently described by Trosvik and de Muinck [25]. They employed ecological concepts of keystone and foundation taxa, which complement their utility as biomarkers. Designation as keystone and foundation taxa requires knowledge of their causative role in a community, whereas biomarkers only need to demonstrate a correlation with diet, lifestyle, or disease state.

We have demonstrated that the "enterotypes" of the human gut microbiome do not represent recurrent microbial communities across the diversity of human populations, nor do they represent two distinguishable communities when Bacteroides and Prevotella are removed from the analysis. We propose that the dominant taxa associated with the original "enterotypes" hypothesis, Bacteroides and Prevotella, as well as other taxa such as Bifidobacterium, Fusobacterium, and various Clostridiales genera, are more accurately understood as biomarkers. The many correlative studies of the gut microbiome and associations with diet, environment, and disease can be made more useful by moving beyond basic ordination plots to rigorously assessing their sensitivity and specificity as biomarkers of lifestyle and disease in clinical subpopulations.

\section{Methods \\ Data compilation}

The data for this article were compiled from six previous studies. We used processed reads for each sample from the Yatsunenko et al. [11] study that were downloaded from the MG-RAST website (http://metagenomics.anl. gov) under the index qiime:850, MG-RAST IDs: 4489349.3 - 4489926.3. The reads were assigned taxonomy by comparison against the SILVA database [26] using GAST [27]. For all other studies, the data source was a table of taxonomic abundances for each sample. We received the Ou et al. [10] data directly from the authors. We downloaded the Human Microbiome Project (HMP) data from the VAMPS website [28] at the Marine Biological Laboratory (https://vamps.mbl.edu) under the name HMP_ST_v3v5. We downloaded the Arumugam et al. [1] datasets from the authors' website (http:// www.bork.embl.de/Docu/Arumugam_et_al_2011/downlo ads.html) under the "individual" subcategory of "Genus and phylum abundance tables of the three datasets." We downloaded the Tyakht et al. [12] study data from the Russian Metagenome Project website (http://www.metagenome.ru/files/rus_met/).

The genera represented in the taxonomy tables were filtered to include only genera whose maximum relative abundance across all samples was greater than 0.0001 or $0.01 \%$. We also removed children under the age of 13 from the Yatsunenko et al. data as all other samples from the combined studies were adult, and we did not want to add childhood as a confounding variable. The summary of the data we received and the modifications we made for each statistical test can be found in supplemental materials (Additional file 4: Table S1). The taxonomic assignments made by each study were consistent with the NIH Human Microbiome Project and standard 
$16 \mathrm{~S}$ databases with consistent taxonomic assignments, allowing the comparison of taxa across the various studies [29].

Lozupone et al. [30] analyzed the effectiveness of meta-study comparisons of human gut samples. Their reanalysis of the data, using OTUs (which they referred to as "species-level"), reproduced the results of the source publications. They specifically demonstrated that differences between Western and agrarian samples, along with age and geography, were ample to outweigh differences in study protocols. In some cases, however, they could discern study-specific clustering of samples from healthy Western guts, but the percent explained by their first two PCoA axes was only $12.5 \%$ combined. We chose to use genus-level taxonomy rather than OTUs; the human gut taxa are well-represented in the $16 \mathrm{~S}$ reference databases, the databases are consistent, and genus is a coarser-grained analysis, and therefore less sensitive than $97 \%$ OTUs to differences in study design. We also note that the great abundance of research on the human gut microbiome using next-generation sequencing techniques is built on the presumption of consistent genus-level results across studies providing an increasing body of information on the nature of both healthy and dysbiotic human gut communities.

\section{Separation into microbial communities}

We evaluated several methods for separating communities into their respective mixture components. Bacteroides is generally seen at a range of abundances including low, medium, and high abundances $[2,3,10]$. Prevotella, on the other hand, can have a bimodal behavior, with either a large relative abundance or almost none at all, especially in smaller studies [14, 15]. Roager et al. [16] found that the ratio of the logarithm of Prevotella to Bacteroides abundance generated a natural and distinct split between Prevotella- and Bacteroides-dominated microbial communities in their samples caused by the bimodal Prevotella distribution [5]. By compiling multiple studies, we uncovered a full range of Prevotella abundance, albeit the intermediate range abundances being less common (Additional file 11: Figure S3). The combined data therefore did not have a natural break in relative abundance values, and the log ratio method was ineffective. We chose instead to simply define Prevotella-dominated and Bacteroides-dominated samples as any sample in which either Prevotella or Bacteroides was the most abundant taxon. All remaining samples were classified together as "Other." We introduced the Prevotella ratio as the ratio of Prevotella abundance to the combined abundance of Prevotella and Bacteroides, to measure the Prevotella abundance relative to Bacteroides in the samples (a value of 0 is no Prevotella and all Bacteroides, and a value of 1 is all Prevotella and no
Bacteroides). This Prevotella ratio provides a continuous variable for measuring the spectrum of abundance values, rather than a binary value representing only the dominant taxon.

\section{Ordination}

We performed both NMDS and PCoA with Bray-Curtis, Morisita-Horn, and Jensen-Shannon Divergence metrics (ordinate and plot_ordination functions in the phyloseq R package [18], and the ellipse package). See Figure S10 in Additional file 10 for the resulting ordination plots. Bray-Curtis was computed on the relative abundance. As expected, all methods provided similar results and we report only the PCoA results in the main paper (Fig. 1) and include the NMDS plots in the Additional files 12 and 13 (Figures S6 and S8 show the screeplots of the eigenvalues/percentage of variances explained). Plot points were labeled according to their most dominant taxa-Bacteroides, Prevotella, or other (Fig. 1a), by their population group within each study (Fig. 1c), or by their Prevotella ratio (defined above) (Fig. 1b). To illustrate the impact of the dominant taxon on the ordination of the microbial communities, we removed both Prevotella and Bacteroides rows in all the data and reran the ordination, without rescaling, to illustrate the change in separation (Fig. 1d).

\section{Correlations and differential abundance analyses}

The number of DNA reads returned by next-generation sequencing for a bacterial sample does not reflect the absolute abundance of bacteria in the original sample. The total number of reads for each sample is effectively arbitrary and therefore represents only the relative not the actual abundance of DNA sequences. Unfortunately, relative abundance data are not statistically sufficient to accurately assess internal microbiome interactions [18], but as several of the studies only provided these proportions and not the original read numbers, we proceeded using relative abundance and took several precautions to avoid the biases this can induce. Since relative abundance values must always tally to $100 \%$, also known as compositional data, values within a sample are not independent and can lead to false negative correlations with parametric methods such as Pearson's correlation. Nonparametric methods, such as Spearman's correlation, are preferred in these types of analyses. We used the Spearman nonparametric method in R [31] (function cor.test) on the relative abundance matrices of the Prevotelladominated and Bacteroides-dominated samples from all studies. We then confirmed our findings using SparCC which was developed by Friedman et al. [17] specifically to evaluate correlations within compositional data and remove false correlations through randomized bootstrap trials. We used SparCC with default parameters, 
generating 100 bootstrap files on all samples. For the studies for which sequence counts were available, the Malawi, Venezuela, and American study [11] and the HMP study of healthy Americans [15], we ran both SparCC [32] and nbinomTest of DESeq2 package [18] on the count data. To include datasets with only relative abundance data in the SparCC analysis, we multiplied the relative abundances by 10,000 to normalize sample counts to 10,000 reads, as per the recommendations of the SparCC authors. The choice of 10,000 meant that relative abundances as low as $0.0001(0.01 \%)$ were included. While converting from relative abundances to integer values of read counts would bias taxa that had a low abundance in samples that had been rarefied, none of the methods of the original studies included rarefaction. The correlations were run on the Prevotella-dominated and Bacteroides-dominated samples for each individual study independently and then on the compiled group of all studies. In all three methods, we defined statistically significant correlations as having a positive correlation coefficient and a multiple-experiments adjusted $p$ value of less than or equal to 0.05 using the Benjamini-Hochberg algorithm (function p.adjust in R). We defined biologically significant correlations useful for developing a microbial community definition to be between community members with a mean abundance $\geq 0.02(2 \%)$ and a prevalence of $75 \%$ (present in at least $75 \%$ of the samples at $\geq 0.01$ relative abundance. Bacteria with lower abundances or prevalences may perform important functions in the gut; they are not informative in a defining the overall community structure.

For the proportionality analysis, the Lovell et al. [19] proposed proportionality algorithm was applied to a table containing all the relative abundances for all the data sets with a modification to accommodate the large number of zero values in this very sparse data. Zero values were replaced by randomly generated uniform values between $10^{\wedge}(-8)$ and $2.10^{\wedge}(-8)$. Values that had a resulting phi value $<0.01$ were considered significant.

\section{Availability of supporting data}

The data and the $\mathrm{R}$ script will be available at a permanent url for the html files associated to the article on publication (see the Rmd and html files have been deposited at the permanent url http://purl.stanford.edu/fs506ff9976).

\section{Endnotes}

${ }^{1}$ Combining "enteric" from the Greek word for intestine, and the English word "type" meaning category. Here, we use the term as originally intended.

${ }^{2} \mathrm{PCoA}$ and MDS are abbreviations for Principal Coordinates Analysis and Multidimensional Scaling, two names for the same ordination method which aims to provide low dimensional Euclidean representations of multivariate data for which a specific distance has been measured.

${ }^{3}$ NMDS designates non-metric multidimensional scaling, a non-parametric version of MDS that aims to provide a Euclidean graphical representation of the data points where the distances between points have the same order as those in the original data.

\section{Additional files}

Additional file 1: Figure S1. PCoA plots using the Bray distance metric with all the samples in the study colored by the origin of the data. (PDF $39 \mathrm{~kb}$ )

Additional file 2: Figure S10. PCoA plot with all the samples except the Yatsunenko study colored by the most prominent taxon. A) Distance calculated using the Jensen-Shannon divergence (JSD) and B) distance calculated using Morisita-Horn. (PDF $17 \mathrm{~kb}$ )

Additional file 3: Figure S7. PCOA plots using the Bray distance metric with only the Yatsunenko study. A) Samples colored by their most prominent taxon. If the sample is dominated neither by Prevotella nor Bacteroides, it is classified as other. B) Samples are colored by their value for the Prevotella ratio (relative abundance of Prevotella/[Bacteroides + Prevotella]) on a spectrum with red indicating no Prevotella and purple no Bacteroides. C) Samples are colored by population of origin. D) The Bray distance has been recalculated without the relative abundances of Bacteroides and Prevotella. Samples are colored by most prominent taxon in the original samples distributions (same as in plot A). (PDF 27 kb)

Additional file 4: Table S1. Summary of the data received for each data set, as well as the format in which the data was used for the multivariate and proportionality analyses. (DOCX $81 \mathrm{~kb}$ )

Additional file 5: RDATAand RMD.tar.gz is a zipped file of all the data and $\mathrm{R}$ markdown files to reproduce all the analyses done in the article and is the files can be found at : <http://purl.stanford.edu/fs506ff9976>. (ZIP $11504 \mathrm{~kb}$ )

Additional file 6: Figure S2. Bacteroides distributions within each population. The sample quantiles are represented on the $x$-axis and all samples are plotted on the same graph. The black line represents all the samples scaled together on the $x$-axis. The green points are from the Native African vs. African American Ou et al. study, the yellow points are from the Russian Urban vs Rural Tyakht et al. study, the pink points are from the Malawi, Venezuela, US Yatsunenko et al. study, the red points are from the Mixed Europe and Asia Arumugam et al. study, the blue points are from the European Arumugam et al. study, and the light blue points are from the NIH Human Microbiome Project study. (PDF 19 kb)

Additional file 7: Figure S11. Boxplot of the top 20 taxa across studies not including the Yatsunenko study. The dark horizontal line represents the mean relative abundance and the box represents the bounds of the 25th and 75th percentiles. (PDF $25 \mathrm{~kb}$ )

Additional file 8: Figure S4. NMDS plots using the Bray distance metric with all the samples except for the Yatsunenko study samples. A) Samples colored by their most prominent taxon. If the sample is dominated neither by Prevotella nor Bacteroides, it is classified as other. B) Samples are colored by their value for the Prevotella ratio (relative abundance of Prevotella/[Bacteroides + Prevotella]) on a spectrum with red indicating no Prevotella and purple no Bacteroides. C) Samples are colored by population of origin. D) The Bray distance has been recalculated without the relative abundances of Bacteroides and Prevotella. Samples are colored by most prominent taxon in the original samples distributions (same as in plot A). (PDF $46 \mathrm{~kb}$ )

Additional file 9: Figure S5. A) Same PCoA plot using the Bray distance metric as in the main paper, except looking at the 3rd and 4th axis, with samples colored by their most prominent taxa. B) Same MDS plot as in A, but with samples colored based on their value for the Prevotella ratio on a spectrum with red indicating no Prevotella and purple no Bacteroides. C) Same PCoA plot with samples colored based on 
population of origin. D) PCoA plot using the Bray distance metric with the Bacteroides and Prevotella relative abundances taken out. Colored by most prominent taxa in the samples before the removal of Prevotella and Bacteroides. (PDF $47 \mathrm{~kb}$ )

Additional file 10: Figure S9. NMDS plots using the Bray distance metric with only the Yatsunenko study. A) Samples colored by their most prominent taxon. If the sample is dominated neither by Prevotella nor Bacteroides, it is classified as other. B) Samples are colored by their value for the Prevotella ratio (relative abundance of Prevotella/[Bacteroides + Prevotella]) on a spectrum with red indicating no Prevotella and purple no Bacteroides. C) Samples are colored by population of origin. D) The Bray distance has been recalculated without the relative abundances of Bacteroides and Prevotella. Samples are colored by most prominent taxon in the original samples distributions (same as in plot A). (PDF $28 \mathrm{~kb}$ )

Additional file 11: Figure S3. Prevotella distributions within each population. The $x$-axis represents the sample quantiles and all the samples are plotted on the same graph. The black line represents all the samples scaled together on the $x$-axis. The green points are from the Native African vs. African American Ou et al. study, the yellow points are from the Russian Urban vs Rural Tyakht et al. study, the pink points are from the Malawi, Venezuela, US Yatsunenko et al. study, the red points are from the Mixed Europe and Asia Arumugam et al. study, the dark blue points are from the European Arumugam et al. study, and the light blue points are from the NIH Human Microbiome Project study. (PDF $16 \mathrm{~kb}$ )

Additional file 12: Figure S6. Relative variances explained by the PCOA axes from the PCoA analyses. A) PCoA variances explained by the axes used in the Fig. $1 a, b$ and $c$ and Figure S5ABC. B) PCoA variances axes used in the adjusted sample plots in Fig. 1d and Figure S5D, which had its Bacteroides and Prevotella relative abundances removed. (PDF $4 \mathrm{~kb}$ )

Additional file 13: Figure S8. Relative variances explained by the PCoA axes from the PCoA analyses A) Relative variances explained by the PCoA axes used in the Additional file 7: Figure S7ABC. B) Relative variances explained by the PCoA axes used in the adjusted sample plot in Additional file 7: Figure S7D, which had its Bacteroides and Prevotella relative abundances removed. (PDF $4 \mathrm{~kb}$ )

\section{Competing interests}

Susan Holmes has a short-term consulting agreement with Second Genome. The authors declare that they have no competing interests.

\section{Authors' contributions}

AG helped with the design of the study, carried out the statistical analysis of the data, and drafted the manuscript. SPH helped with the design of the study, carried out the statistical analysis of the data, and edited the manuscript. SMH conceived and designed the study and edited the manuscript. All authors read and approved the final manuscript.

\section{Authors' information}

Anastassia Gorvitovskaia is a senior at Brown University working towards a Bachelor of Science in Computational Biology.

Susan Holmes is a Professor of Statistics at Stanford University, and her team has developed the phyloseq toolbox for analyzing microbiome data within R. Susan Huse was Assistant Professor (Research) at Brown University at the time the study was conducted and is now a Senior Computational Scientist at Symbiota, a biotech company working on applications of the plant microbiome in agriculture.

\section{Acknowledgements}

We gratefully acknowledge Stephen O'Keefe for having shared data tables from his study on cancer in rural Africans and African Americans with us and the Richard Fox of the Josephine Bay Paul Center at the Marine Biological Laboratory for providing computational systems support. AG was funded by a Summer Research Assistantship grant for summer 2014. SPH was funded by NIH R01 GM099534.

\section{Author details}

'Brown University, Providence, RI, USA. ${ }^{2}$ Sequoia Hall, Stanford University, Stanford, CA, USA. Indigo Agriculture Inc, Cambridge, MA, USA.
Received: 16 November 2015 Accepted: 2 March 2016 Published online: 12 April 2016

\section{References}

1. Arumugam M, Raes J, Pelletier E, Le Paslier D, Yamada T, Mende DR, et al. Enterotypes of the human gut microbiome. Nature. 2011;473(7346):174-80. http://www.nature.com/nature/journal/v473/n7346/full/nature09944.html.

2. Wu GD, Chen J, Hoffmann C, Bittinger K, Chen Y-Y, Keilbaugh SA, et al. Linking long-term dietary patterns with gut microbial $E=e n t e r o t y p e s$. Science. 2011;334(6052):105-8. doi:10.1126/science.1208344.

3. Huse SM, Ye Y, Zhou Y, Fodor AA. A core human microbiome as viewed through 16S rRNA sequence clusters. PLoS One. 2012;7(6):e34242. doi:10. 1371/journal.pone.0034242.

4. Jeffery IB, Claesson MJ, OToole PW, Shanahan F. Categorization of the gut microbiota: enterotypes or gradients? Nat Rev Micro. 2012;10(9):591-2.

5. Koren O, Knights D, Gonzalez A, Waldron L, Segata N, Knight R, et al. A guide to enterotypes across the human body: meta-analysis of microbial community structures in human microbiome datasets. PLoS Comput Biol. 2013:9(1):e1002863. doi:10.1371/journal.pcbi.1002863.

6. Knights D, Ward TL, McKinlay CE, Miller H, Gonzalez A, McDonald D, et al. Rethinking "Enterotypes". Cell Host \& Microbe. 2014;16(4):433-7. http://dx. doi.org/10.1016/j.chom.2014.09.013.

7. Zhou Y, Mihindukulasuriya K, Gao H, La Rosa P, Wylie K, Martin J, et al. Exploration of bacterial community classes in major human habitats. Genome Biology. 2014;15(5):R66.

8. De Filippo C, Cavalieri D, Di Paola M, Ramazzotti M, Poullet JB, Massart S, et al. Impact of diet in shaping gut microbiota revealed by a comparative study in children from Europe and rural Africa. Proceedings of the National Academy of Sciences. 2010;107(33):14691-6. doi:10.1073/pnas.1005963107.

9. Sela DA, Mills DA. Nursing our microbiota: molecular linkages between bifidobacteria and milk oligosaccharides. Trends in Microbiology. 2010;18(7):298-307. doi:10.1016/j.tim.2010.03.008.

10. Ou J, Carbonero F, Zoetendal EG, DeLany JP, Wang M, Newton K, et al. Diet, microbiota, and microbial metabolites in colon cancer risk in rural Africans and African Americans. The American Journal of Clinical Nutrition. 2013:98(1):111-20.

11. Yatsunenko T, Rey FE, Manary MJ, Trehan I, Dominguez-Bello MG, Contreras $M$, et al. Human gut microbiome viewed across age and geography. Nature. 2012;486(7402):222-7. http://www.nature.com/nature/journal/v486/n7402/ full/nature11053.html.

12. Tyakht AV, Kostryukova ES, Popenko AS, Belenikin MS, Pavlenko AV, Larin AK et al. Human gut microbiota community structures in urban and rural populations in Russia. Nat Commun. 2013;4. doi:10.1038/ncomms3469

13. Tyakht AV, Alexeev DG, Popenko AS, Kostryukova ES, Govorun VM. Rural and urban microbiota: to be or not to be? Gut Microbes. 2014;5(3):351-6. http:// dx.doi.org/10.4161/gmic.28685.

14. Arumugam M, Raes J, Pelletier E, Le Paslier D, Yamada T, Mende DR et al. Enterotypes of the human gut microbiome. Nature 473: 174-180 doi:10. 1038/nature09944. http://www.nature.com/nature/journal/v473/n7346/full/ nature09944.html

15. Consortium THMP. Structure, function and diversity of the healthy human microbiome. Nature. 2012;486(7402):207-14. http://www.nature.com/nature/ journal/v486/n7402/full/nature11234.html.

16. Roager HM, Licht TR, Poulsen SK, Larsen TM, Bahl MI. Microbial enterotypes, inferred by the prevotella-to-bacteroides ratio, remained stable during a 6-month randomized controlled diet intervention with the New Nordic Diet. Applied and Environmental Microbiology. 2014;80(3):1142-9. doi:10.1128/aem.03549-13.

17. Friedman J, Alm EJ. Inferring correlation networks from genomic survey data. PLoS Comput Biol. 2012;8(9):e1002687. doi:10.1371/journal.pcbi. 1002687.

18. McMurdie PJ, Holmes S. Waste not, want not: why rarefying microbiome data is inadmissible. PLoS Comput Biol. 2014;10(4):e1003531. doi:10.1371/ journal.pcbi.1003531.

19. Lovell D, Pawlowsky-Glahn V, Egozcue JJ, Marguerat S, Bähler J. Proportionality: a valid alternative to correlation for relative data. PLoS Comput Biol. 2015;11(3):e1004075. doi:10.1371/journal.pcbi.1004075.

20. Pearson K. Mathematical contributions to the theory of evolution. On a form of spurious correlation which may arise when indices are used in the measurement of organs. Proceedings of the Royal Society of London. 1896; 60(359-367):489-98. doi:10.1098/rspl.1896.0076. 
21. Group BDW. Biomarkers and surrogate endpoints: preferred definitions and conceptual framework. Clinical Pharmacology and Therapeutics. 2001;69(3):89-95.

22. David LA, Maurice CF, Carmody RN, Gootenberg DB, Button JE, Wolfe BE, et al. Diet rapidly and reproducibly alters the human gut microbiome. Nature. 2014; 505(7484):559-63. doi:10.1038/nature12820. http://www.nature.com/nature/ journal/v505/n7484/full/nature12820.html.

23. Baxter NT, Zackular JP, Chen GY, Schloss PD. Structure of the gut microbiome following colonization with human feces determines colonic tumor burden. Microbiome. 2014;2:20. doi:10.1186/2049-2618-2-20.

24. Kostic AD, Gevers D, Pedamallu CS, Michaud M, Duke F, Earl AM, et al. Genomic analysis identifies association of Fusobacterium with colorectal carcinoma. Genome Research. 2012;22(2):292-8. doi:10.1101/gr.126573.111.

25. Trosvik $\mathrm{P}$, de Muinck EJ. Ecology of bacteria in the human gastrointestinal tract-identification of keystone and foundation taxa. Microbiome. 2015;3: 44. doi:10.1186/s40168-015-0107-4.

26. Quast C, Pruesse E, Yilmaz P, Gerken J, Schweer T, Yarza P, et al. The SILVA ribosomal RNA gene database project: improved data processing and web-based tools. Nucleic Acids Research. 2013;41(D1):D590-D6.

27. Huse SM, Dethlefsen L, Huber JA, Mark Welch D, Relman DA, Sogin ML. Exploring microbial diversity and taxonomy using SSU rRNA hypervariable tag sequencing. PLoS Genetics. 2008:4(11):e1000255.

28. Huse S, Mark Welch D, Voorhis A, Shipunova A, Morrison H, Eren A, et al. VAMPS: a website for visualization and analysis of microbial population structures. BMC Bioinformatics. 2014;15(1):41.

29. Liu Z, DeSantis TZ, Andersen GL, Knight R. Accurate taxonomy assignments from 165 rRNA sequences produced by highly parallel pyrosequencers. Nucleic Acids Research. 2008;36(18):e120. doi:10.1093/nar/gkn491.

30. Lozupone CA, Stombaugh J, Gonzalez A, Ackermann G, Wendel D, VázquezBaeza Y, et al. Meta-analyses of studies of the human microbiota. Genome Research. 2013;23(10):1704-14. doi:10.1101/gr.151803.112.

31. R Core Team. R: a language and environment for statistical computing. Vienna, Austria: R Foundation for Statistical Computing; 2012.

32. McMurdie PJ, Holmes S. phyloseq: an R package for reproducible interactive analysis and graphics of microbiome census data. 2013:e61217.

\section{Submit your next manuscript to BioMed Central and we will help you at every step:}

- We accept pre-submission inquiries

- Our selector tool helps you to find the most relevant journal

- We provide round the clock customer support

- Convenient online submission

- Thorough peer review

- Inclusion in PubMed and all major indexing services

- Maximum visibility for your research

Submit your manuscript at www.biomedcentral.com/submit

C Biomed Central 